\title{
2-connections, a lattice point of view
}

\author{
B. Bouzid* and M. Tahiri \\ Laboratoire de Physique Théorique d'Oran (LPTO), Université d'Oran, BP 1524 El M'Naouer, \\ 31100 Es-Senia, Oran, Algeria
}

\begin{abstract}
We show that the transition laws for a 2-connection can be recovered by discretizing the base 2-space of a 2-bundle into an Euclidean hypercubic lattice. The aim of this work is to serve as an example of how important results in higher gauge theory, which have been derived in a continuous setting, can also be derived in the lattice scheme.
\end{abstract}

\section{Introduction}

In ordinary gauge theory built upon a $G$-principal bundle $E \stackrel{p}{\rightarrow} B$, a connection $A$ describes parallel transport of point particles along paths. This connection can be locally seen as a $\operatorname{Lie}(G)$-valued 1-form on the base space $B$, hence it associates a group element $\operatorname{hol}_{A}(\gamma) \in G$ to each path $\gamma$ of the space, called the holonomy of $A$ along $\gamma$. In this way, group elements become associated to paths of the space. We call a configuration or coloring of this space a given choice of such associations. In lattice gauge theory, the base space is discretized into an Euclidean hypercubic lattice with lattice spacing $a$, physical laws are recovered by taking the limit $a \rightarrow 0$ (see e.g. [11] and references therein).

On the other hand, transformations of extended objects like strings cannot be described using such a connection, since strings move along surfaces, whereas point particles move along paths, gauge theories need to be extended to include connections that can describe parallel transport of both point particles and strings along paths and surfaces respectively. There will certainly be some interplay between these two kinds of transformations, and this should be handled by the extended theory. This theory is called higher gauge theory [1] and is the extension of gauge theory in the langage of higher category theory, which is well suited to deal with such problems. Higher gauge theory is based on generalizations of spaces, groups, bundles and connections to, respectively, 2spaces, 2-groups, 2-bundles and 2-connections using the so-called enrichment and internalization processes.

Our main goal is to give a simple example of application of lattice technics to higher gauge theory in order to recover the transformation laws for a 2-connection. Although these laws have already been derived in a continuous setting [12,13, formulating higher gauge theory on a lattice has its own benefit: it may be applicable to computer-based numerical simulations (see e.g. [10] and references therein). Let us recall that passing to the lattice formalism has proven to be a crucial

*bouzid.badreddine@gmail.com 
step for the computer-based numerical simulations of gauge theories in the past (see e.g. 9] and references therein).

Higher lattice gauge theory involves associating not only group elements to links of the lattice, but also to its plaquettes. Then, using relations inherited from higher category theory, important results that lie at the heart of higher gauge theory can be recovered. In the present paper, we use some of the techniques that appeared in 7, but which have initially been introduced in 8 . However, we perform a slight modification in the aforementioned techniques. First, we note that point-wise transformations have been represented by the authors as if they were propagating over the lattice. We remark that this choice leads to a trucated form of the transformation laws. This is mainly due to the higher order $\varepsilon$ terms that vanish, where $\varepsilon$ is what they called the "height" of the point-wise transformation. Instead, we describe transformations propagating on the space (such as the holonomies) by assigning group elements to links and plaquettes, wherease point-wise transformations are described by the assignment of group elements to vertices.

\section{Definitions and notations}

We descretize the trivial smooth base 2-space $B$ into an Euclidean lattice $B=a \mathbb{Z} \times a \mathbb{Z} \times \cdots \times a \mathbb{Z}$. Let $e_{\mu}$ denotes the unit vector in the $\mu$ direction, the vector $a e_{\mu}$ will be written $\mu$ for short. A link $\gamma_{x \rightarrow \mu}$ stands for the oriented path between the ordered pair of points $(x, x+\mu)$

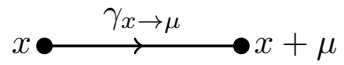

whereas a plaquette $\Sigma_{x}^{\mu \rightarrow \nu}$ stands for the oriented surface with boundary the ordered quadruple $(x, x+\mu, x+\mu+\nu, x+\nu)$

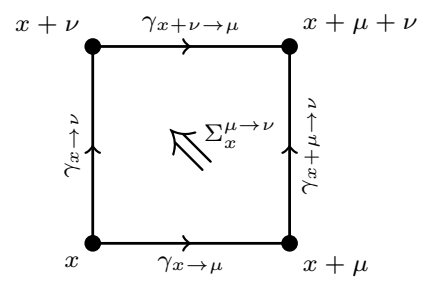

All quantities in the lattice written without argument will be understood as being evaluated at the origin $x=0$. All groups considered in this paper are matrix groups.

The holonomy of the 2-connection for the patch $U_{i}$ along a link at the origin propagating in the $\mu$ direction $\gamma_{0 \rightarrow \mu}$ will be denoted

$$
\operatorname{hol}_{i \mu}=e^{\int_{\gamma_{0 \rightarrow \mu}} A_{i}}
$$

The holonomy of the 2-connection for the patch $U_{i}$ on a plaquette at the origin propagating in the $\mu$ and $\nu$ directions $\Sigma_{0}^{\mu \rightarrow \nu}$ will be denoted

$$
\operatorname{hol}_{i \mu \nu}=e^{\int_{\Sigma_{0}^{\mu \rightarrow \nu}} B_{i}}
$$


The 2 -group $\mathcal{G}$ will be seen as a 2 -category with a single object denoted $\star$. On each patch $U_{i}$, there is a 2 -groupoid $\mathcal{P}_{2}\left(U_{i}\right)$ of thin homotopy classes of smooth lazy paths and surfaces [1, the holonomy is then a 2 -functor

$$
\operatorname{hol}_{i}: \mathcal{P}_{2}\left(U_{i}\right) \rightarrow \mathcal{G}
$$

that takes each point of $U_{i}$ to the single object $\star$ of $\mathcal{G}$

$$
\operatorname{hol}_{i}^{(0)}(x)=\star
$$

The 1-morphism map of the holonomy functor

$$
\operatorname{hol}_{i}^{(1)}: 1 \operatorname{Mor}\left(\mathcal{P}_{2}\left(U_{i}\right)\right) \rightarrow \mathcal{G}^{(1)}
$$

acts on origin-based links of the lattice as

$$
\operatorname{hol}_{i}^{(1)}\left(0 \bullet \stackrel{\gamma_{0 \rightarrow \mu}}{\longrightarrow} \bullet \mu\right)=\star \stackrel{\mathrm{hol}_{i \mu}}{\longrightarrow} \star
$$

Although the images of all lattice vertices are always $\star$, for the sake of clarity we will write hol $_{i}^{(0)}(x)=x$ for each vertex $x$

$$
\operatorname{hol}_{i}^{(1)}\left(0 \bullet \stackrel{\gamma_{0 \rightarrow \mu}}{\longrightarrow} \bullet \mu\right)=0 \bullet \stackrel{\operatorname{hol}_{i \mu}}{\longrightarrow} \bullet \mu
$$

But keeping in mind that, if seen as living in $\mathcal{G}$, all vertices of this diagram are the single object $\star$, while if it is seen as living in $\mathcal{P}_{2}\left(U_{i}\right)$, labels on links are the coloring of the lattice.

In the same spirit, the 2-morphism map of the holonomy functor

$$
\operatorname{hol}_{i}^{(2)}: 2 \operatorname{Mor}\left(\mathcal{P}_{2}\left(U_{i}\right)\right) \rightarrow \mathcal{G}^{(2)}
$$

acts on origin-based plaquettes of the lattice as

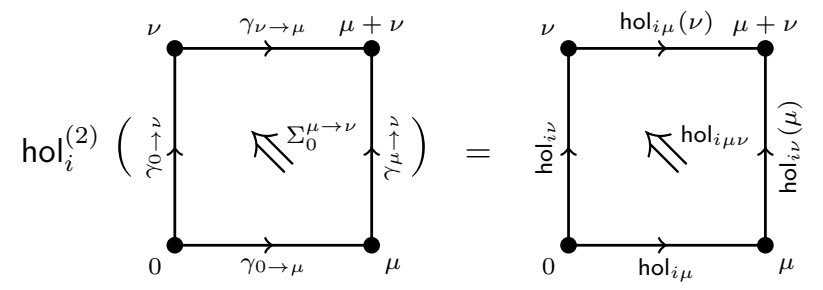

\section{2-connections}

Let $E \stackrel{p}{\rightarrow} B$ be a $\mathcal{G}$-2-bundle, where $\mathcal{G}$ is some (strict) smooth 2-group corresponding to the Lie crossed module $(G, H, t, \alpha)$ and $(\mathfrak{g}, \mathfrak{h}, d t, d \alpha)$ be its differential crossed module [2, 3, 12]. We choose $B$ to be a trivial smooth 2-space equipped with an ordinary cover $\left\{U_{i}\right\}_{i \in I}$ which is hypercubic-wise, i.e., the opens $U_{i}$ are open hypercubes. This will ensure that no links and no plaquettes are partially included in some opens $U_{i}$, except perhaps for their boundaries. But we remark that if an endpoint 
of a link does not belong to the patch $U_{i}$ that contains the rest of the link, the integration is not going to differ much from that using the whole link. The transition functions on the cover $\left\{U_{i}\right\}_{i \in I}$ are $g_{i j}, h_{i j k}$ and $k_{i}$. We will also restrict ourselves to the case $k_{i}=1$. On each patch $U_{i}$ the local holonomy 2 -functor hol $_{i}$ is specified by two differential forms

$$
\begin{array}{ll}
A_{i} & \in \Omega^{1}\left(U_{i}, \mathfrak{g}\right) \\
B_{i} & \in \Omega^{2}\left(U_{i}, \mathfrak{h}\right)
\end{array}
$$

such that the fake curvature vanishes

$$
F_{A_{i}}+d t\left(B_{i}\right)=0
$$

where $F_{A_{i}}$ is the curvature 2 -form of $A_{i}$.

The transition pseudonatural isomorphism $g_{i j}:$ hol $_{i} \Rightarrow \mathrm{hol}_{j}$ is specified by the transition functions $g_{i j}$ together with differential forms $a_{i j} \in \Omega^{1}\left(U_{i j}, \mathfrak{h}\right)$, whereas the modification $h_{i j k}: g_{i j} g_{j k} \Rightarrow$ $g_{i k}$ is specified by the transition functions $h_{i j k}$, such that on every double overlap $U_{i j}$ the following transformation laws hold

$$
\begin{aligned}
& A_{i}=g_{i j} A_{j} g_{i j}^{-1}+g_{i j} \mathrm{~d} g_{i j}^{-1}-\mathrm{d} t\left(a_{i j}\right) \\
& B_{i}=\alpha\left(g_{i j}\right)\left(B_{j}\right)+\mathrm{d} a_{i j}+a_{i j} \wedge a_{i j}+\mathrm{d} \alpha\left(A_{i}\right) \wedge a_{i j}
\end{aligned}
$$

and on every triple overlap $U_{i j k}$ the following transformation law holds

$$
a_{i j}+\alpha\left(g_{i j}\right) a_{j k}=h_{i j k}^{-1} a_{i k} h_{i j k}+h_{i j k}^{-1} \mathrm{~d} h_{i j k}+h_{i j k}^{-1} \mathrm{~d} \alpha\left(A_{i}\right) h_{i j k}
$$

Let us recall that these transformation laws have already been derived in [12,13, using a continuous setting. Here we use a different approach which has been inspired from [7]. There, the authors used lattice calculus to recover the fake curvature connection (which has also been previously derived in a continuous setting) as well as other important results.

\section{Transition laws for the 2-connection}

In higher lattice gauge theory, transition functions are represented not only by 1-morphisms

$$
\forall x \in \mathrm{Ob}\left(\mathcal{P}_{2}\left(U_{i j}\right)\right): g_{i j}(x) \in \mathcal{G}^{(1)}
$$

but also by 2-morphisms

$$
\forall \gamma \in 1 \operatorname{Mor}\left(\mathcal{P}_{2}\left(U_{i j}\right)\right): g_{i j}(\gamma) \in \mathcal{G}^{(2)}
$$

such that if $\gamma: x \rightarrow y$, we have

$$
g_{i j}(\gamma): \operatorname{hol}_{i}(\gamma) g_{i j}(y) \Rightarrow g_{i j}(x) \operatorname{hol}_{j}(\gamma)
$$

To derive equation (3.1) we take a link $\gamma_{0 \rightarrow \mu} \in U_{i j}$, its images in $\mathcal{G}$ via the 2-connections $\left(A_{i}, B_{i}\right)$ and $\left(A_{j}, B_{j}\right)$ are related by the transition functions as follows

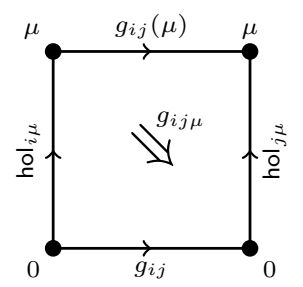


The 2-morphism $g_{i j \mu}$ has $\operatorname{hol}_{i \mu} g_{i j}(\mu)$ as source 1-morphism and $g_{i j}$ hol $_{j \mu}$ as target 1-morphism, we have then the following relation which stems directly from higher category theory [1]

$$
g_{i j} \operatorname{hol}_{j \mu}=t\left(g_{i j \mu}\right) \operatorname{hol}_{i \mu} g_{i j}(\mu)
$$

thus hol $i \mu$ is

$$
\begin{aligned}
\text { hol }_{i \mu} & =t\left(g_{i j \mu}\right)^{-1} g_{i j} \text { hol }_{j \mu} g_{i j}(\mu)^{-1} \\
e^{\int_{\gamma_{0} \rightarrow \mu} A_{i}} & =t\left(g_{i j \mu}^{-1}\right) g_{i j} e^{\int_{\gamma_{0 \rightarrow \mu}} A_{j}} g_{i j}(\mu)^{-1}
\end{aligned}
$$

The differential forms $a_{i j}$ describe the transition pseudonatural isomorphisms $g_{i j}$ at the plaquette level, thus

$$
g_{i j \mu}=e^{a a_{i j \mu}}
$$

Hereafter, as $a \rightarrow 0$ the symbol $\approx$ means that we approximate the equalities by neglecting terms of order higher than the dimension we are working on.

As $a \rightarrow 0$ the connection can be considered as constant along each link, so that we get

$$
e^{\int_{\gamma_{0 \rightarrow \mu}} A_{i}} \approx e^{a A_{i \mu}}
$$

On the other hand, using a Taylor expansion in $g_{i j}(\mu)$ and the derivative of $t$ we finally get

$$
e^{a A_{i \mu}} \approx e^{-a \mathrm{~d} t\left(a_{i j \mu}\right)} g_{i j} e^{a A_{j \mu}}\left(g_{i j}^{-1}+a \partial_{\mu} g_{i j}^{-1}\right)
$$

Again, using Taylor expansions of exponentials, we get

$$
\begin{aligned}
1+a A_{i \mu} & \approx\left(1-a \mathrm{~d} t\left(a_{i j \mu}\right)\right) g_{i j}\left(1+a A_{j \mu}\right)\left(g_{i j}^{-1}+a \partial_{\mu} g_{i j}^{-1}\right) \\
& \approx 1+a\left(g_{i j} \partial_{\mu} g_{i j}^{-1}+g_{i j} A_{j \mu} g_{i j}^{-1}-\mathrm{d} t\left(a_{i j \mu}\right)\right)
\end{aligned}
$$

We thus recover (3.1).

Now to derive equation (3.2) we take a plaquette at the origin, its images in $\mathcal{G}$ via the 2connections $\left(A_{i}, B_{i}\right)$ and $\left(A_{j}, B_{j}\right)$ are related by the transition functions as follows

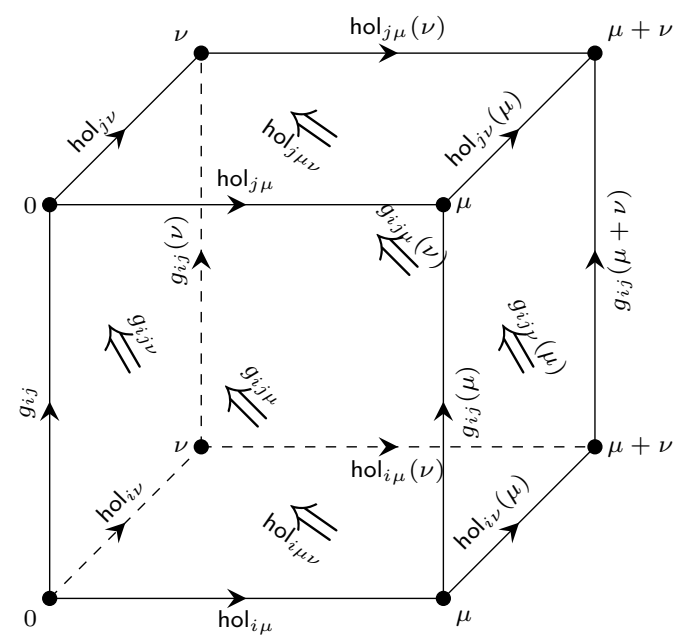


The coloring 2-morphism $\operatorname{hol}_{i \mu \nu}$ has source hol ${ }_{i \mu} \operatorname{hol}_{i \nu}(\mu)$ and target hol ${ }_{i \nu} \operatorname{hol}_{i \mu}(\nu)$, it sweeps the bottom side of the cube. Alternatively, it can also be seen as sweeping the remaining sides of the cube since the diagram commutes in $\mathcal{G}$.

Let us denote horizontal (vertical) composition of 2-morphisms by $o_{h}\left(\circ_{v}\right)$.

We note that the horizontal composition of a 2-morphism with a 1-morphism is a shortcut of the horizontal composition of this 2-morphism with identity 2-morphism of the 1-morphism, that is, for example for $g \in G$ and $h \in H$

$$
h \circ_{h} g:=h \circ_{h} 1_{g}
$$

We remark first that

$$
\operatorname{hol}_{i \mu \nu} \circ_{h} g_{i j}(\mu+\nu)=\underset{\sim}{\longrightarrow} \uparrow=
$$

Now to write down the other expression of that 2-morphism (i.e. when it sweeps the remaining sides), we will need the following pieces of the cube

$$
\operatorname{hol}_{i \mu} \circ_{h} g_{i j \nu}(\mu)=
$$

Using the following equality,

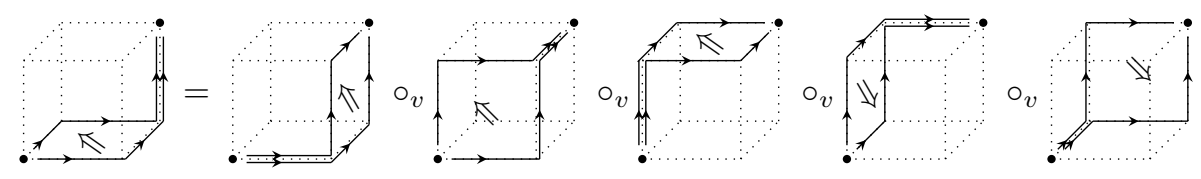


we get

$$
\begin{aligned}
\operatorname{hol}_{i \mu \nu} & =\left[\operatorname{hol}_{i \nu} \triangleright g_{i j \mu}^{-1}(\nu)\right] g_{i j \nu}^{-1}\left[g_{i j} \triangleright \text { hol }_{j \mu \nu}\right] g_{i j \mu}\left[\operatorname{hol}_{\mathrm{i} \mu} \triangleright g_{i j \nu}(\mu)\right] \\
e^{\int_{\Sigma_{0}^{\mu \rightarrow \nu} B_{i}}} & =\alpha\left(e^{\int_{\gamma_{0} \rightarrow \nu} A_{i}}\right)\left(g_{i j \mu}^{-1}(\nu)\right) g_{i j \nu}^{-1} \alpha\left(g_{i j}\right)\left(e^{\int_{\Sigma_{0}^{\mu} \rightarrow \nu} B_{j}}\right) g_{i j \mu} \alpha\left(e^{\int_{\gamma_{0} \rightarrow \mu} A_{i}}\right)\left(g_{i j \nu}(\mu)\right)
\end{aligned}
$$

Again, as $a \rightarrow 0$ the 2-connection can be considered as constant on each plaquette, so that

$$
e^{\int_{\Sigma_{0}^{\mu \rightarrow \nu} B_{i}}} \approx e^{a^{2} B_{i \mu \nu}}
$$

Using the derivative of $\alpha$ and a Taylor expansion on $g_{i j \mu}(\nu)$ we get

$$
e^{a^{2} B_{i \mu \nu}} \approx e^{a \mathrm{~d} \alpha\left(A_{i \nu}\right)}\left(e^{-a a_{i j \mu}-a^{2} \partial_{\nu} a_{i j \mu}}\right) e^{-a a_{i j \nu}} \alpha\left(g_{i j}\right)\left(e^{a^{2} B_{j \mu \nu}}\right) e^{a a_{i j \mu}} e^{a \mathrm{~d} \alpha\left(A_{i \mu}\right)}\left(e^{a a_{i j \nu}+a^{2} \partial_{\mu} a_{i j \nu}}\right)
$$

Expanding exponentials and after some calculations we get

$$
\begin{gathered}
1+a^{2} B_{i \mu \nu} \approx\left(1+a \mathrm{~d} \alpha\left(A_{i \nu}\right)\right)\left(1-a a_{i j \mu}-a^{2} \partial_{\nu} a_{i j \mu}\right)\left(1-a a_{i j \nu}\right) \\
\alpha\left(g_{i j}\right)\left(1+a^{2} B_{j \mu \nu}\right)\left(1+a a_{i j \mu}\right)\left(1+a \mathrm{~d} \alpha\left(A_{i \mu}\right)\right)\left(1+a a_{i j \nu}+a^{2} \partial_{\mu} a_{i j \nu}\right) \\
\approx \begin{array}{c}
1+a^{2}\left[\alpha\left(g_{i j}\right)\left(B_{j \mu \nu}\right)+\partial_{\mu} a_{i j \nu}-\partial_{\nu} a_{i j \mu}+a_{i j \mu} a_{i j \nu}-a_{i j \nu} a_{i j \mu}\right. \\
+ \\
\left.+\mathrm{d} \alpha\left(A_{i \mu}\right)\left(a_{i j \nu}\right)-\mathrm{d} \alpha\left(A_{i \nu}\right)\left(a_{i j \mu}\right)\right]
\end{array}
\end{gathered}
$$

where we have dropped out the symmetric terms. So we recover (3.2).

It is worth noting that in [7 the authors wrote pointwise gauge transformations as they were propagating in an $\alpha$ direction over a link of length $\varepsilon$. However, it turns out that with such a choice, transformations like $g_{i j \mu}(\nu)$ would have been

$$
g_{i j \mu}(\nu) \approx e^{\varepsilon a a_{i j \mu}+\varepsilon a^{2} \partial_{\nu} a_{i j \mu}}
$$

and then, the $a_{i j} \wedge a_{i j}$ term would have disappeared from the transformation laws because of their $a^{2} \varepsilon^{2}$ order.

Finally to derive equation (3.3) we take the triangle that represents the action of the modification $h_{i j k}$ on transition functions, its images in $\mathcal{G}$ via the 2 -connections $\left(A_{i}, B_{i}\right)$ and $\left(A_{j}, B_{j}\right)$ are related by the transition functions as follows

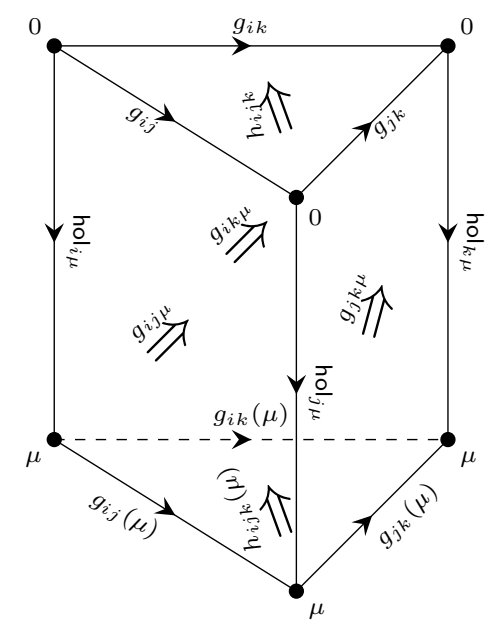


The coloring 2-morphism $g_{i k \mu}$ has source hol ${ }_{i \mu} g_{i k}(\mu)$ and target $g_{i k}$ hol $_{k \mu}$, it sweeps the backside face of the prism. Alternatively, it can be seen as sweeping the remaining sides of the prism since the diagram commutes in $\mathcal{G}$. Let us repeat the previous steps for this diagram.

We remark first that

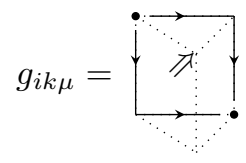

Now we write down the other expression of that 2-morphism

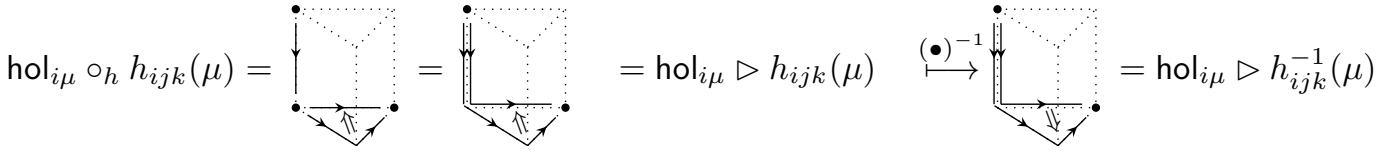

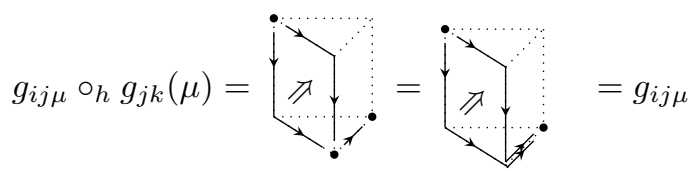

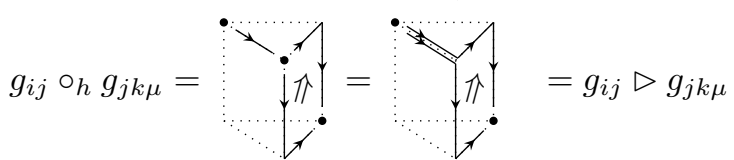

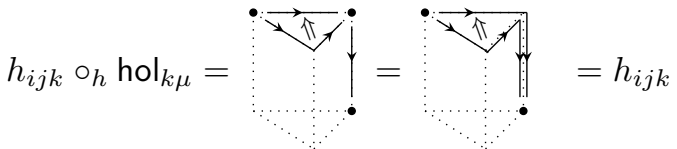

Using the following equality

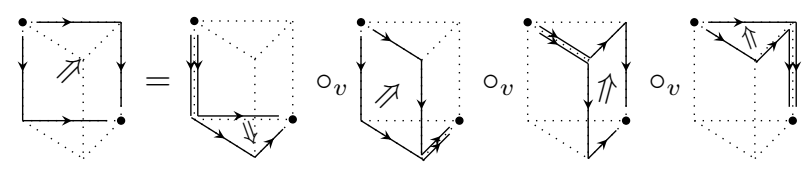

we get

$$
\begin{aligned}
g_{i k \mu} & =h_{i j k}\left[g_{i j} \triangleright g_{j k \mu}\right] g_{i j \mu}\left[\operatorname{hol}_{i \mu} \triangleright h_{i j k}^{-1}(\mu)\right] \\
h_{i j k}^{-1} g_{i k \mu}\left[\operatorname{hol}_{i \mu} \triangleright h_{i j k}(\mu)\right] & =\left[g_{i j} \triangleright g_{j k \mu}\right] g_{i j \mu}
\end{aligned}
$$

The R.H.S is

$$
\begin{aligned}
{\left[g_{i j} \triangleright g_{j k \mu}\right] g_{i j \mu} } & =\left(\alpha\left(g_{i j}\right) g_{j k \mu}\right) g_{i j \mu} \\
& =\left(\alpha\left(g_{i j}\right) e^{a a_{j k \mu}}\right) e^{a a_{i j \mu}}
\end{aligned}
$$

Using Taylor expansions of exponentials we get

$$
\begin{aligned}
{\left[g_{i j} \triangleright g_{j k \mu}\right] g_{i j \mu} } & \approx\left(\alpha\left(g_{i j}\right)\left(1+a a_{j k \mu}\right)\right)\left(1+a a_{i j \mu}\right) \\
& \approx 1+a\left(a_{i j \mu}+\alpha\left(g_{i j}\right) a_{j k \mu}\right)
\end{aligned}
$$


The L.H.S is

$$
h_{i j k}^{-1} g_{i k \mu}\left[\operatorname{hol}_{i \mu} \triangleright h_{i j k}(\mu)\right]=h_{i j k}^{-1} g_{i k \mu}\left[\alpha\left(e^{a A_{i \mu}}\right) h_{i j k}(\mu)\right]
$$

Using Taylor expansions of exponentials and of $h_{i j k}(\mu)$, as well as the derivative of $\alpha$ we get

$$
\begin{aligned}
h_{i j k}^{-1} g_{i k \mu}\left[\operatorname{hol}_{i \mu} \triangleright h_{i j k}(\mu)\right] & \approx h_{i j k}^{-1}\left(1+a a_{i k \mu}\right)\left[\left(1+a \mathrm{~d} \alpha\left(A_{i \mu}\right)\right)\left(h_{i j k}+a \partial_{\mu} h_{i j k}\right)\right] \\
& \approx 1+a\left(h_{i j k}^{-1} a_{i k \mu} h_{i j k}+h_{i j k}^{-1} \partial_{\mu} h_{i j k}+h_{i j k}^{-1} \mathrm{~d} \alpha\left(A_{i \mu}\right) h_{i j k}\right)
\end{aligned}
$$

This leads us to obtain (3.3).

\section{Conclusion and outlook}

We have shown that calculus on the lattice can systematically be used to derive important results of higher gauge theory. This is achieved by coloring plaquettes in addition to links without any other assumption. The physical laws can be recovered from the $a \rightarrow 0$ limit. However, some coherence relations between group elements coloring links and group elements coloring plaquettes are pivotal for this construction. These relations prove to be at the heart of the higher category theory: the first group elements are morphisms (or 1-morphisms) of some 2-group, while the latter are 2-morphisms between these morphisms. This is reminiscent of what happens in some gauge theories where symmetries between symmetries appear due to the presence of second class constraints. It is worth noting that in $B F$ theory, which is known to have this kind of metasymmetries, and which has close relations to quantum gravity [4, the gauge fields defining the theory form a 2-connection [6].

All this tends to prove that higher category theory is a fertile ground where theories can be enriched, by systematically extending basic structures underlying them using the two main tools of higher category theory: internalization and enrichment [3].

Finally, let us point out that what we have called 2-bundles is also known under the name of gerbes, and that a whole theory of differential gerbes already exists [5]. It can be interesting to approach some aspects of this theory using calculus on lattice defined in the present article, for example, to found the more general transformation laws when no $k_{i}=1$ restriction is made.

\section{References}

[1] John C Baez and John Huerta. An invitation to higher gauge theory. General Relativity and Gravitation, 43(9):2335-2392, 2011.

[2] John C Baez and Urs Schreiber. Higher gauge theory: 2-connections on 2-bundles. arXiv preprint hep-th/0412325, 2004.

[3] John C Baez and Urs Schreiber. Higher gauge theory. Contemporary Mathematics, 431:7-30, 2007.

[4] B Bouzid and M Tahiri. From bf models to quantum gravity. In THE 8TH INTERNATIONAL CONFERENCE ON PROGRESS IN THEORETICAL PHYSICS (ICPTP 2011), volume 1444, pages 149-153. AIP Publishing, 2012.

[5] Lawrence Breen and William Messing. Differential geometry of gerbes. Advances in Mathematics, 198(2):732-846, 2005. 
[6] Francesco Cianfrani. The kinematical hilbert space of loop quantum gravity from bf theories. Classical and Quantum Gravity, 28(17):175014, 2011.

[7] Florian Girelli and Hendryk Pfeiffer. Higher gauge theory - differential versus integral formulation. Journal of mathematical physics, 45(10):3949-3971, 2004.

[8] Harald Grosse and Karl-Georg Schlesinger. Duals for non-abelian lattice gauge theories by categorical methods. International Journal of Theoretical Physics, 40(2):459-475, 2001.

[9] Noboru Kawamoto, Noriaki Sato, and Yukiya Uchida. 4-dimensional bf gravity on the lattice. Nuclear Physics B, 574(3):809-848, 2000.

[10] Arthur E. Lipstein and Ronald A. Reid-Edwards. Lattice gerbe theory. Journal of High Energy Physics, 2014(9):34, 2014.

[11] Yuri Makeenko. Methods of contemporary gauge theory. Cambridge University Press, 2002.

[12] Urs Schreiber. From loop space mechanics to nonabelian strings. arXiv preprint hepth/0509163, 2005.

[13] Urs Schreiber and Konrad Waldorf. Smooth functors vs. differential forms. Homology, Homotopy and Applications, 13(1):143-203, 2011. 